\title{
Simulated Field Maps: Toward Improved Susceptibility Artefact Correction in Interventional MRI
}

\author{
Martin Kochan ${ }^{\star 1}$, Pankaj Daga ${ }^{1}$, Ninon Burgos ${ }^{1}$, Mark White ${ }^{2}$, \\ M. Jorge Cardoso ${ }^{1,3}$, Laura Mancini ${ }^{2}$, Gavin P. Winston ${ }^{4}$, \\ Andrew W. McEvoy ${ }^{2}$, John Thornton ${ }^{2}$, Tarek Yousry ${ }^{2}$, John S. Duncan ${ }^{4}$, \\ Danail Stoyanov ${ }^{1}$ and Sebastien Ourselin ${ }^{1,3}$ \\ ${ }^{1}$ Centre for Medical Image Computing, \\ University College London, London, UK \\ ${ }^{2}$ National Hospital for Neurology and Neurosurgery, \\ UCLH NHS Foundation Trust, London, UK \\ ${ }^{3}$ Dementia Research Centre, Institute of Neurology, \\ University College London, London, UK \\ ${ }^{4}$ Department of Clinical and Experimental Epilepsy, Institute of Neurology, \\ University College London, London, UK
}

\begin{abstract}
Intraoperative MRI is a powerful modality for acquiring structural and functional images of the brain to enable precise imageguided neurosurgery. In this paper, we propose a novel method for simulating main magnetic field inhomogeneity maps during intraoperative MRI-guided neurosurgery. Our method relies on an air-tissue segmentation of intraoperative patient specific data, which is used as an input to a subsequent field simulation step. The generated simulation can then be used to enhance the precision of image-guidance. We report results of our method on 12 patient datasets acquired during image-guided neurosurgery for anterior lobe resection for surgical management of focal temporal lobe epilepsy. We find a close agreement between the field inhomogeneity maps acquired as part of the imaging protocol and the simulated field inhomogeneity maps generated by the proposed method.
\end{abstract}

Keywords: image-guided neurosurgery, interventional MRI, inhomogeneity field map simulation

\section{Introduction}

Anterior temporal lobe resection (ATLR) is an effective treatment for refractory temporal lobe epilepsy. However, resective surgery may result in severe complications such as contralateral superior visual field deficit (VFD) that restricts the seizure-free patient from returning to regular activity. Magnetic resonance imaging (MRI) is the preferred modality for imaging soft-tissue brain morphology and

^m.kochan.12@ucl.ac.uk 
function for diagnosis and postoperative follow-up. Additionally, interventional MRI (iMRI) can potentially be used to enhance the precision of pathological tissue resection while minimizing the damage to healthy brain structures. By preserving critical brain tissues, the patients may benefit from improved outcomes and quality of life.

Image-guided neurosurgery for ATLR is an established surgical specialisation but localization accuracy can be adversely affected by intraoperative physiomechanical deformation of the soft tissue, generally referred to as brain shift, which can be caused by cerebrospinal fluid (CSF) drainage, tissue retraction, brain swelling and the resection itself [1]. Imaging using iMRI can provide valuable information about the anatomy, which can be used to compensate for brain shift by registering preoperative and intraoperative images. Recently, Daga et al. [2] have proposed multimodal co-registration of anatomical T1-weighted MRI images paired with fractional anisotropy maps (DWI-FA) derived from diffusionweighted imaging (DW-MRI) image sets, as a means of estimating brain shift. This approach takes into account the locations of white matter tracts that are not discernible visually nor on the T1-weighted anatomical scans. However, DWMRI image sets are acquired using the echo planar imaging (EPI) MRI pulse sequence, which suffers from severe geometric distortion, caused by the very limited acquisition bandwidth of EPI in the phase-encode (PE) dimension of the image. Severe distortion occurs in EPI images even due to small inhomogeneity in the main magnetic field $B_{0}$ on the order of several ppm.

The first source of $B_{0}$ inhomogeneity is due to hardware constraints and can be reduced (shimmed) to several ppm by means of superconducting shim coils [3]. The second source of $B_{0}$ field inhomogeneity is due to perturbation of the magnetic field by non-uniform geometric distribution of magnetic susceptibility in the imaged volume. This inhomogeneity is largest near air-tissue boundaries, such as the sinuses, the petrous part of the temporal bone [4], and the resection cavity itself. The susceptibility-related inhomogeneity is shimmed using a set of room-temperature (RT) shim coils. However, imperfect shimming and higher-order perturbations result in residual inhomogeneity. The distortion of the EPI image associated with this residual inhomogeneity is called the susceptiblity artefact. A popular approach to correct for the susceptibility artefact is to acquire the residual inhomogeneity field maps using a specific MR pulse sequence [4]. However, the acquired inhomogeneity field maps differ from the true field maps due to low SNR near air-tissue boundaries (e.g. the resection margin) and due to MR signal dropout (e.g. close to head-holder attachment pins) [5]. In iMRI guided neurosurgery, the diversions from true field maps can adversely affect image guidance accuracy.

In this paper, we propose to simulate a field map from T1-weighted and T2weighted iMRI images acquired as part of a standard iMRI scanning protocol. Previously, Jenkinson et al. [6] demonstrated a perturbation method to calculate a $B_{0}$ inhomogeneity field from air-tissue segmentation derived from computed tomography (CT) images. Poynton et al. [5] demonstrated that non-surgical T1weighted images can be segmented into air and tissue classes using a probabilistic 
$\mathrm{CT}$ atlas, and reported that a subsequent application of the method [6] results in close overall agreement between the acquired and simulated field maps. However, we observe that a probabilistic atlas is not suited to the segmentation of intraoperative iMR images that contain air-filled craniotomy and resection areas of variable shape that depend on the surgery and patient morphology. Instead, we employ an expectation-maximization (EM) based segmentation method informed by priors derived from a synthetic CT image. We compute the synthetic $\mathrm{CT}$ from the intraoperative T1-weighted image and a database of MR/CT pair templates. We subsequently feed the air-tissue segmentation into the method [6]. The field map simulation is evaluated by comparison with field maps acquired during iMRI guided ATLR neurosurgery for 12 cases. The proposed method generates field maps in close agreement with the acquired field maps.

This result has the potential to lead to improvements in EPI image correction and image guidance for neurosurgery. Additionally, the proposed method can also be used to correct distortion in historical intraoperative EPI datasets, which did not include field map acquisition as part of the acquisition protocol.

\section{Methods}

\subsection{Field map in terms of voxel displacement}

Let the magnetic field at point $\boldsymbol{x}$ be $B_{0}+\Delta B_{0}(\boldsymbol{x})[\mathrm{T}]$ where $B_{0}$ is the homogeneous field and $\Delta B_{0}(\boldsymbol{x})$ is the inhomogeneity field map, which can be equivalently expressed as $\gamma \Delta B_{0}(\boldsymbol{x})[\mathrm{rad} / \mathrm{s}]$ or $\frac{\gamma \Delta B_{0}(\boldsymbol{x})}{2 \pi}[\mathrm{Hz}]$. For the purposes of image correction, one is interested in the millimetre displacement along the phase encode direction that the inhomogeneity causes to an EPI image. The displacement can be calculated based on theory in $[4,7]$. Consider the acquisition of a single EPI slice with matrix size $N \times N$ and voxel dimensions $r_{\mathrm{FE}}$ in the frequency encode (FE) direction and $r_{\mathrm{PE}}$ in the phase encode (PE) direction, respectively. The EPI slice is reconstructed by the inverse Fourier transform of the MR signal. In the PE direction, the MR signal sampling rate is $\frac{N}{T_{\text {acq }}}[\mathrm{Hz}]$, where $T_{\text {acq }}$ is the signal acquisition time. The reconstructed image resolution in the PE direction is $\frac{N}{N T_{\text {acq }}}=\frac{1}{T_{\text {acq }}}[\mathrm{Hz} / \mathrm{pixel}]$ or $T_{\text {acq }}[\mathrm{pixel} / \mathrm{Hz}]$. Since the $\mathrm{PE}$ gradient is used to encode position along the PE direction, the above offset corresponds to a distortion along the $\mathrm{PE}$ direction of size:

$$
d_{\mathrm{PE}}(\boldsymbol{x})=\frac{\gamma \Delta B_{0}(\boldsymbol{x})}{2 \pi} T_{\mathrm{acq}} r_{\mathrm{PE}} .
$$

In this study, the EPI image correction itself was only performed for visual confirmation (Figure 2), by converting the field map into a vector displacement/deformation vector field and subsequently resampling the image using cubic spline interpolation [8].

\subsection{Field map acquisition}

Field map acquisition was based on the method introduced in [4], whereby the field map is dependent on phase difference map between the phase components of 
MR images acquired during two MR signal echoes, separated by echo difference time $T_{\mathrm{ED}}$. The phase difference corresponds to spin phase evolution during $T_{\mathrm{ED}}$ but is modulo- $2 \pi$ phase-wrapped due to unknown number of elapsed revolutions. Additionally, the phase difference signal is noisy in low spin-density areas (air and bone) and has low SNR near air-tissue boundaries. Therefore, to recover the inhomogeneity $\gamma \Delta B_{0}(\boldsymbol{x})$, we used a novel phase-unwrapping algorithm based on a probabilistic model spatially constrained by means of a Markov random field (MRF) formulation, as presented in [8]. We de-meaned the recovered phase difference map, since the recovered phase difference necessarily has an arbitrary constant component.

\subsection{Air-tissue segmentation}

The magnetic susceptibility values for soft tissue $\left(\approx-9.1 \times 10^{-6}\right)$ and bone $\left(\approx-11.4 \times 10^{-6}\right)$ are similar, but both are significantly different from that of air $\left(\approx 0.4 \times 10^{-6}\right)[5]$. Therefore, we need a binary labelling of the head into tissue and air. For each subject, a segmentation was performed on the sum of the T1and T2-weighted iMRI image (a pseudo spin density image). In this image, the soft tissues (grey and white matter, the eyes) were grey, CSF and fat tissue were bright, and air and bone were black.

For the air-tissue segmentation, we used a segmentation algorithm based on an expectation-maximization (EM) intensity model spatially regularized using an MRF [9]. Tissue was segmented into three partial volume classes: air, bone and soft tissue (Figure 1, centre right) and later the bone and soft tissue classes were combined into the tissue class. Each class had its associated spatial prior map. The spatial prior maps were calculated from a closed skull synthetic CT. In $\mathrm{CT}$, each of the 3 classes has a unique intensity range and therefore, the $\mathrm{CT}$ was intensity-transformed using 2 sigmoid functions that acted as separators to select tissue based on intensity. During the EM segmentation, full MRF strength was chosen to enforce the presence of air in the resection area (as opposed to soft tissue) and of air in the craniotomy area (as opposed to bone).

The closed skull synthetic CT image was constructed from the T1-weighted iMRI image following the method described by Burgos et al. [10]. The method relies on a database consisting of 6 pairs of co-registered T1-weighted MR / CT images from healthy subjects. Each MR image from the database was non-rigidly registered to the intraoperative iMRI image so that each CT could be propagated into the iMRI space. The resampled CT images were fused together using a voxel-wise rank-based weighting scheme (Figure 1, centre left).

The intraoperative field of view contains the cranial part of the head, but does not include the head below the nose level. The later field map simulation step (as described in Section 2.4) assumes that no tissue is present outside of the air-tissue segmentation volume that has a significant contribution to the field distribution inside the volume. Therefore, an approximated lower head tissue volume was constructed in a volume inferior to the iMRI (Figure 1, right). To construct the lower head tissue volume, the affine registration from the CT synthesis step was reused to resample the MR templates into the target volume, 
and the resampled volumes intensity transformed using a sigmoid function and averaged.
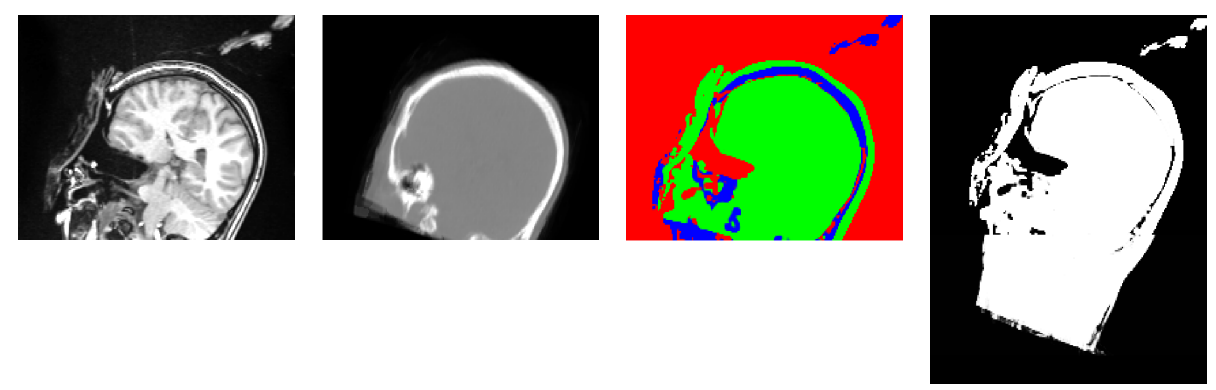

Fig. 1. Air-tissue segmentation. Left: a T1-weighted intraoperative image. The section runs through a plane close to the anatomical coronal plane (head at angle due to intraoperative orientation). Middle left: an accompanying synthetic CT. Middle right: the result of the segmentation (red for air, green for soft-tissue, blue for bone). Right: the final air-tissue segmentation (black for air, white for tissue) with the fitted lower head volume.

\subsection{Field map estimation}

The field map estimation follows from [6] and models the first order perturbations of the main magnetic field. The susceptibility $\chi$ can be expanded as $\chi=\chi_{0}+\delta \chi_{1}$, where $\chi_{0}$ is the magnetic susceptibility of air, $\delta$ is the susceptibility difference between air and brain tissue and $\chi_{1}$ is a binary variable describing the tissue type. The first order perturbations of the z-component of the main magnetic field $\left(B_{z}^{1}\right)$ can be written in terms of the main magnetic field $\left(B_{z}^{0}\right)$ :

$$
B_{z}^{1}=\frac{\chi_{1}}{3+\chi_{0}} B_{z}^{0}-\frac{1}{1+\chi_{0}}\left(\left(\frac{\partial^{2} G}{\partial z^{2}}\right) *\left(\chi_{1} B_{z}^{0}\right)\right)
$$

where $G$ is the Green's function $G(x)=(4 \pi r)^{-1}$ and $r=\sqrt{x^{2}+y^{2}+z^{2}}$. Note that the expression is simplified considerably due to the fact that we only have a non-zero component in the longitudinal axis (z-direction) of the main magnetic field.

The convolution $H(\boldsymbol{x})$ for a single voxel with the resolution $(a, b, c)$ for a constant field along the $\mathrm{z}$-axis is given by:

$$
H(\boldsymbol{x})=\left(\frac{\partial^{2} G}{\partial z^{2}}\right) *\left(\chi_{1} B_{z}^{0}\right)=\sum_{i, j, k \in(-1,1)}(i j k) F\left(x+\frac{i a}{2}, y+\frac{j b}{2}, z+\frac{k c}{2}\right)
$$

where $F(\boldsymbol{x})=\frac{1}{4 \pi} \arctan \left(\frac{x y}{z r}\right)$. 
Due to the linearity of Equation (2), the single voxel solutions can be added together to compute the total field:

$$
B_{z}^{1}(\boldsymbol{x})=\sum_{\boldsymbol{x}^{\prime}} \chi_{1} \boldsymbol{x}^{\prime} H\left(\boldsymbol{x}-\boldsymbol{x}^{\prime}\right)
$$

where $\boldsymbol{x}^{\prime}$ are the voxel locations and $\boldsymbol{x}$ is the point where the field is evaluated. This can be implemented using the 3D Fast Fourier Transform.

Although this approach simulates the field distribution due to the main coil, MRI scanners also contain room-temperature (RT) shim coils, whose purpose is to decrease the inhomogeneity in the imaged volume. The RT shim coils are wound to form magnetic fields that follow first- and second-order spherical harmonics, $S(\boldsymbol{x})=\left[x, y, z, z^{2}-\left(x^{2}+y^{2}\right) / 2, x z, y z, x^{2}-y^{2}, 2 x y\right](\boldsymbol{x})$, where $\boldsymbol{x}=\mathbf{0}$ at the magnet isocentre [11]. The field in the scanner becomes $B_{z}^{1}(\boldsymbol{x})-S \boldsymbol{\theta}$, where the coefficients $\boldsymbol{\theta}=\left[\theta_{1}, \theta_{2}, \ldots, \theta_{8}\right]^{T}$ are proportional to the currents in the shim coils, which are dynamically optimized by the scanner during image acquisition based on the field in the imaged volume [11]. In this simulation, we approximate the shim currents as a linear combination that minimizes the inhomogeneity field across the field of view, as used in [5]. We perform a least-squares fit of the spherical harmonics to determine $\hat{\boldsymbol{\theta}}=\operatorname{argmin}_{\boldsymbol{\theta}}\left(B_{z}^{1}(\boldsymbol{x})-\boldsymbol{S} \boldsymbol{\theta}\right)$.

\section{Results}

The proposed algorithm was validated on 12 datasets that were acquired using interventional MRI during ATLR procedures. Validation was done as part of an audit to assess the usability of simulated field maps in a clinical scenario. The images were acquired using a 1.5T Espree MRI scanner (Siemens, Erlangen, Germany) designed for interventional procedures. The intraoperative protocol included a T1-weighted FLASH image $(\mathrm{TR}=5.25 \mathrm{~ms}$, TE $=2.5 \mathrm{~ms}$, flip angle $=15^{\circ}, 0.547 \times 0.547 \times 1.25 \mathrm{~mm}$ grid of $512 \times 512 \times 176$ voxels $)$ and a T2weighted turbo spin echo image $(\mathrm{TR}=3200 \mathrm{~ms}, \mathrm{TE}=510 \mathrm{~ms}$, flip angle $=$ $120^{\circ}, 1.0 \times 1.0 \times 1.0 \mathrm{~mm}$ grid of $256 \times 256 \times 176$ voxels), a DW-MRI dataset of 65 diffusion-weighted images acquired using a single shot EPI sequence with GRAPPA-based parallel imaging (acceleration factor of $2,2.5 \times 2.5 \times 2.5 \mathrm{~mm}$ grid of $84 \times 84 \times 49$ voxels, readout time $35.52 \mathrm{~ms}$ ) and a field map acquired using a gradient-recalled echo pulse sequence $(2.91667 \times 2.91667 \times 2.9 \mathrm{~mm}$ grid of $72 \times 72 \times 43$ voxels, echo time difference of $4.76 \mathrm{~ms}$ ).

The DW-MRI dataset for each subject was corrected as per Section 2.1 using the acquired field map and the proposed simulated field map, respectively. An example for a subject is shown in Figure 2.

The most direct validation of the simulated field map would be to compare DWI images corrected using acquired and simulated field maps, respectively, against anatomical landmarks identified on the intraoperative T1-weighted images, which are not affected by the susceptibility artefact (Figure 2). However, due to the low resolution and low signal-to-noise ratio of DW-MRI, the landmarks are challenging to identify reliably and repeatably. Since there is no way 

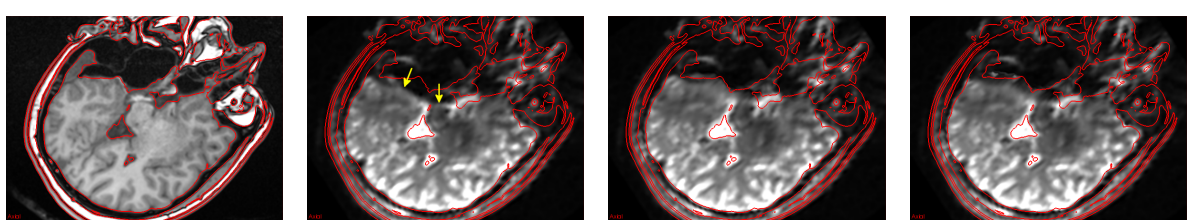

Fig. 2. Detail of correction for the susceptibility artefact. Left: an intraoperative T1weighted image unaffected by the distortion. The section runs through a plane close to the anatomical axial plane (head at angle due to intraoperative orientation). A brain surface outlined using a surface extractor ${ }^{2}$ is shown for reference (red outline). Middle left: an uncorrected "b0" DW-MRI image (an image for which no diffusion gradients are applied). Arrows point at an area of severe susceptibility distortion. Middle right: the "b0" image corrected using the acquired field map. Right: the "b0" image corrected using the simulated field map.

of measuring the true field maps in vivo, we compared the simulated field maps to the acquired field maps (Figure 3). The field maps were expressed in $\mathrm{mm}$ of displacement along the PE direction, as these are the units significant to the correction. Next, we calculated statistics for the difference between the simulated and acquired field maps. The results for the 12 subjects are reported in Table 1. For most of the brain, there is a close agreement. However, the differences follow a long-tailed distribution, so that in some areas, there are larger disagreements.

\begin{tabular}{ccccc}
\hline Mean ( std ) & Median P90 P95 P99 \\
\hline $0.86(1.13)$ & 0.57 & 1.83 & 2.64 & 5.34 \\
$1.16(1.50)$ & 0.68 & 2.68 & 3.78 & 7.15 \\
$0.98(1.37)$ & 0.55 & 2.30 & 3.36 & 6.29 \\
$0.89(1.29)$ & 0.48 & 2.08 & 3.03 & 5.97 \\
$1.00(1.37)$ & 0.63 & 2.16 & 3.19 & 6.24 \\
$0.77(1.03)$ & 0.50 & 1.60 & 2.25 & 4.74 \\
$0.93(1.17)$ & 0.60 & 1.98 & 2.80 & 5.67 \\
$0.94(1.41)$ & 0.49 & 2.12 & 3.21 & 7.06 \\
$1.35(1.84)$ & 0.80 & 2.94 & 4.22 & 9.03 \\
$1.06(1.47)$ & 0.65 & 2.36 & 3.33 & 6.83 \\
$1.23(1.84)$ & 0.60 & 2.99 & 4.33 & 9.50 \\
$0.95(1.39)$ & 0.56 & 2.10 & 3.13 & 6.44 \\
\hline $1.01(1.40)$ & 0.59 & 2.26 & 3.27 & 6.69
\end{tabular}

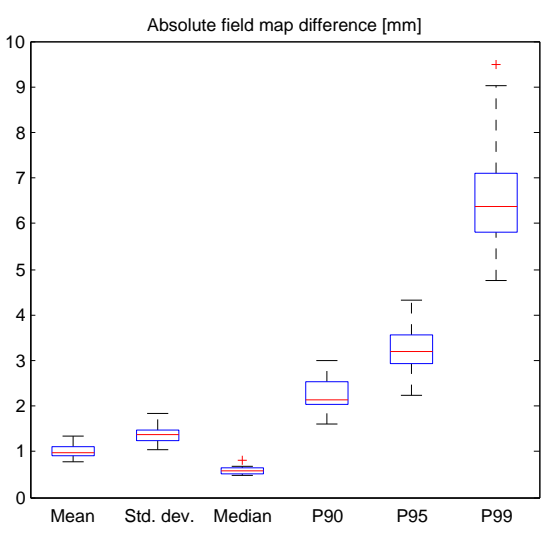

Table 1. Quantification of absolute difference (in $\mathrm{mm}$ ) between the correction displacement in the phase encode direction as predicted by the proposed simulated field map and the acquired field map, respectively, for the 12 subjects. Only voxels within brain mask are considered. The mean, standard deviation, median, and $90^{\text {th }}, 95^{\text {th }}$ and $99^{\text {th }}$ percentile values are reported. The bottom row contains column averages.

\footnotetext{
${ }^{2}$ As included in NiftyView (http://cmic.cs.ucl.ac.uk/home/software).
} 

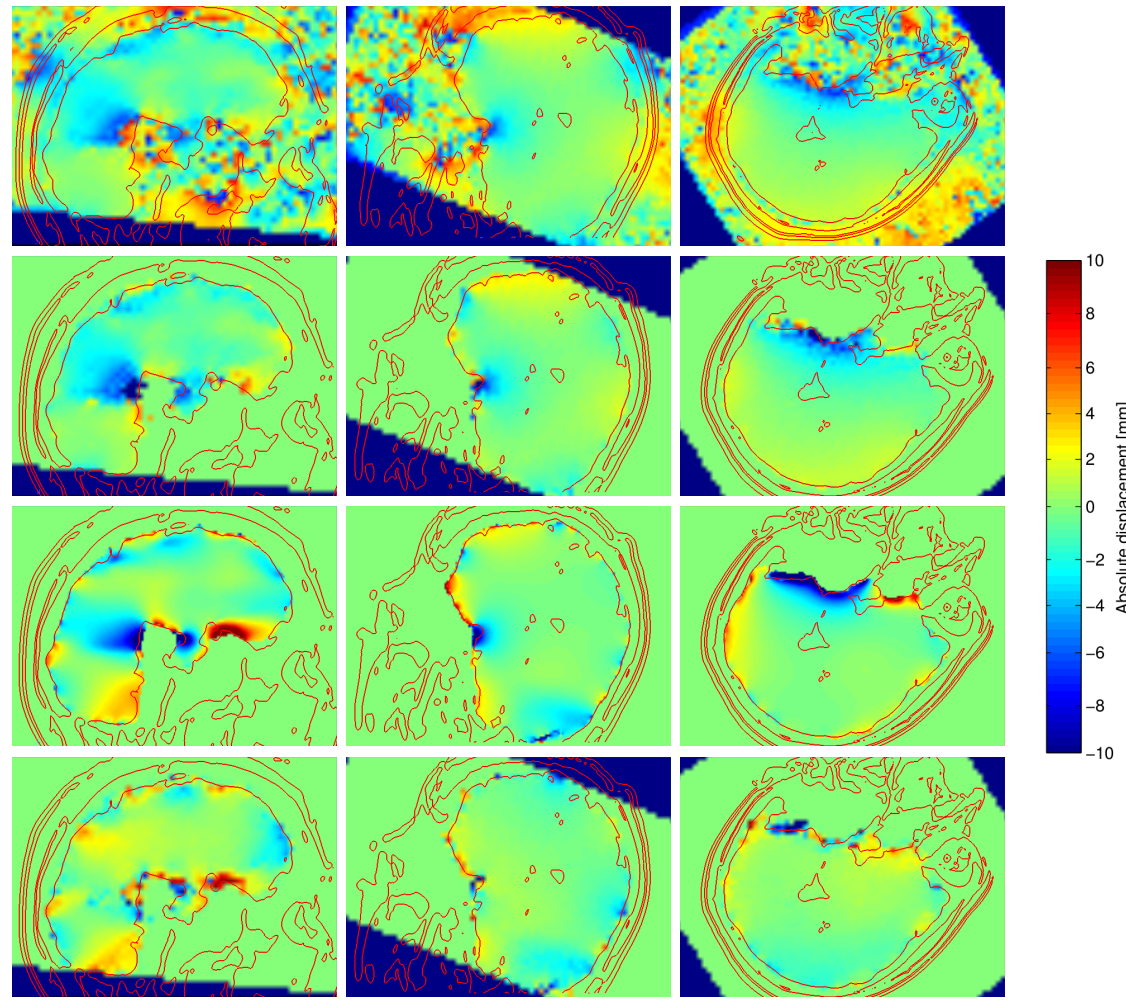

Fig. 3. Field maps expressed as mm of displacement along the phase-encode direction. The view is centered at the resection area of surgery. First row: A phase-wrapped acquired field map for a representative subject, showing a step change in phase value close to the resection margin. Second row: The acquired field map after phase-unwrapping. Only the volume inside the brain mask, as employed by the phase-unwrapping algorithm, is shown. Third row: A corresponding simulated field map (considered only inside the brain mask for fair comparison). Last row: The voxel-wise absolute difference between the simulated and the phase-unwrapped acquired field maps. Left to right: coronal, sagittal and axial sections, not coincident with anatomical planes due to intraoperative orientation of the head. 


\section{Discussion}

Across the subjects, the simulated and acquired field maps on average differ by $1.01 \pm 1.40 \mathrm{~mm}$ in the brain volume. This is within the voxel size of the DWI dataset (2.5 mm, which is typical for DW-MRI datasets). This number also has to be evaluated with respect to a desired resection accuracy, which is patient and surgeon specific and difficult to define. We believe that $1 \mathrm{~mm}$ resection accuracy in areas of low difference is clinically useful. However, since the difference between field maps follows a long-tailed distribution, we attempt to interpret the values of the field maps in areas of more significant difference to deduce where the simulated field maps are more correct, and vice versa.

We observe that the simulated field map is more positive in the vicinity of the resection area. We hypothesize that this could be due to an accumulated error in phase-unwrapping caused by the low SNR in this area and hence due to an underestimated acquired field map.

We observe that near the regions of signal dropout, as visible near the headholder attachment pins, the simulated field map is more positive than the acquired field map. This is in line with the expectation to see a reduced phase evolution in regions of signal dropout and hence due to an underestimated acquired field map.

We also observe that, conversely, near the petrous part of the temporal bone in both hemispheres and anteriorly in the frontal lobe, the simulated field maps are $2-3 \mathrm{~mm}$ above the acquired field maps. This likely occurs because the proposed segmentation method overestimates the size of the air-filled cavities. This overestimation is caused by the high penalty imposed on the bone class in the $\mathrm{EM} / \mathrm{MRF}$ segmentation step, which had been empirically found to be necessary, to robustly segment the craniotomy area as completely air-filled, when relying on the EM/MRF algorithm alone. Therefore, if it was possible to introduce a method to segment the resection cavity and the craniotomy area robustly, the penalty on bone in the the EM/MRF algorithm could be relaxed and the overestimation of the simulated field map could be reduced.

\section{Conclusion}

In summary, field map simulation is important for iMRI guided neurosurgery and in this study we have proposed a method that can achieve a close agreement between the simulated and acquired field maps for 12 patients. We suggest that in the future, simulated field maps could be used to regularize the phaseunwrapping of intraoperatively acquired field maps.

While our results are promising, a significant obstacle for intraoperative use of the proposed method is the computational time required to simulate the field map, currently above 20 minutes (Intel Core i5 @ $3.30 \mathrm{GHz}$ ). Therefore, a possible future work would be to explore methods to speed up the CT synthesis and field map calculation, for instance using GPU hardware. 
Acknowledgements. This work was supported by the UCL Doctoral Training Programme in Medical and Biomedical Imaging studentship funded by the EPSRC. Danail Stoyanov would like to thank for the support of The Royal Academy of Engineering/EPSRC Research Fellowship. Sebastien Ourselin receives funding from the EPSRC (EP/H046410/1, EP/J020990/1, EP/K005278), the MRC (MR/J01107X/1), the EU-FP7 project VPH-DARE@IT (FP7-ICT2011-9-601055), the NIHR Biomedical Research Unit (Dementia) at UCL and the National Institute for Health Research University College London Hospitals Biomedical Research Centre (NIHR BRC UCLH/UCL High Impact Initiative).

\section{References}

1. Nimsky, C., Ganslandt, O., Cerny, S., Hastreiter, P., Greiner, G., Fahlbusch, R.: Quantification of, visualization of, and compensation for brain shift using intraoperative magnetic resonance imaging. Neurosurgery 47.5, 1070-1080 (2000)

2. Daga, P., Winston, G., Modat, M., White, M., Mancini, L., Cardoso, M.J., Symms, M., Stretton, J., McEvoy, A.W., Thornton, J., Micallef, C., Yousry, T., Hawkes, D.J., Duncan, J.S., Ourselin, S.: Accurate Localization of Optic Radiation During Neurosurgery in an Interventional MRI Suite. IEEE Transactions on Medical Imaging 31.4, 882-891 (2012)

3. Clare, S., Evans, J., Jezzard, P.: Requirements for room temperature shimming of the human brain. Magn. Reson. Med. 55, 210-214 (2006)

4. Jezzard, P., Balaban, R.S.: Correction for geometric distortion in echo planar images from b0 field variations. Magn. Reson. Med. 34, 65-73 (1995)

5. Poynton, C., Jenkinson, M., Wells, W.: Atlas-Based Improved Prediction of Magnetic Field Inhomogeneity for Distortion Correction of EPI Data. In: G.-Z. Yang et al. (Eds.): MICCAI 2009, Part II, LNCS vol. 5762, pp. 951-959. Springer, Heidelberg (2009)

6. Jenkinson, M., Wilson J. L., Jezzard, P.: Perturbation method for magnetic field calculations of nonconductive objects. Magnetic Resonance in Medicine 52.3, 471477 (2004)

7. Hutton, C., Bork, A., Josephs, O., Deichmann, R., Ashburner, J., Turner, R.: Image distortion correction in fMRI: a quantitative evaluation. Neuroimage 16.1, 217-240 (2002)

8. Daga, P, Modat, M., Winston, G, White, M., Mancini, L., McEvoy, A. W., Thornton, J., Yousry, T., Duncan, J. S., Ourselin, S.: Susceptibility artefact correction by combining B0 field maps and non-rigid registration using graph cuts. SPIE Medical Imaging, International Society for Optics and Photonics, 86690B-86690B (2013)

9. Cardoso, M. J.: Improved Maximum a Posteriori Cortical Segmentation by Iterative Relaxation of Priors G.-Z. Yang et al. (Eds.): MICCAI 2009, Part II, LNCS 5762, 441-449 (2009)

10. Burgos, N., Cardoso, M. J., Modat, M., Pedemonte, S., Dickson, J., Barnes, A., Duncan, J. S., Atkinson, D., Arridge, S. R., Hutton, B. F., Ourselin, S.: Attenuation Correction Synthesis for Hybrid PET-MR Scanners. K. Mori et al. (Eds.): MICCAI 2013, Part II, LNCS 8150, 147-154 (2013)

11. Gruetter, R., Boesch, C.: Fast, noniterative shimming of spatially localized signals. In vivo analysis of the magnetic field along axes. Journal of Magnetic Resonance, 96.2, 323-334 (1992) 